\title{
Two-Stage Robust Security-Constrained Unit Commitment with Optimizable Interval of Uncertain Wind Power Output
}

\author{
Dayan Sun, ${ }^{1,2}$ Liudong Zhang, ${ }^{3,4}$ Dawei Su, ${ }^{2}$ and Yubo Yuan ${ }^{3}$ \\ ${ }^{1}$ College of Energy and Electrical Engineering, Hohai University, Nanjing, Jiangsu 211100, China \\ ${ }^{2}$ State Grid Jiangsu Electric Power Company, Nanjing, Jiangsu 210024, China \\ ${ }^{3}$ Electric Power Research Institute of State Grid Jiangsu Electric Power Company, Nanjing, Jiangsu 211103, China \\ ${ }^{4}$ School of Electrical Engineering, Southeast University, Nanjing, Jiangsu 210096, China
}

Correspondence should be addressed to Liudong Zhang; zldon_1987@126.com

Received 16 February 2017; Accepted 11 June 2017; Published 20 July 2017

Academic Editor: Fazal M. Mahomed

Copyright (c) 2017 Dayan Sun et al. This is an open access article distributed under the Creative Commons Attribution License, which permits unrestricted use, distribution, and reproduction in any medium, provided the original work is properly cited.

\begin{abstract}
Because wind power spillage is barely considered, the existing robust unit commitment cannot accurately analyze the impacts of wind power accommodation on on/off schedules and spinning reserve requirements of conventional generators and cannot consider the network security limits. In this regard, a novel double-level robust security-constrained unit commitment formulation with optimizable interval of uncertain wind power output is firstly proposed in this paper to obtain allowable interval solutions for wind power generation and provide the optimal schedules for conventional generators to cope with the uncertainty in wind power generation. The proposed double-level model is difficult to be solved because of the invalid dual transform in solution process caused by the coupling relation between the discrete and continuous variables. Therefore, a two-stage iterative solution method based on Benders Decomposition is also presented. The proposed double-level model is transformed into a single-level and twostage robust interval unit commitment model by eliminating the coupling relation, and then this two-stage model can be solved by Benders Decomposition iteratively. Simulation studies on a modified IEEE 26-generator reliability test system connected to a wind farm are conducted to verify the effectiveness and advantages of the proposed model and solution method.
\end{abstract}

\section{Introduction}

At present, the variability, limited predictability, and antipeaking regulation inherent in wind power have created significant challenge to power systems operation with high wind power penetration $[1,2]$. Unit commitment (UC), one of the most crucial processes in power systems schedule and operation, has been evolved from deterministic formulation into uncertainty formulation [3-6] to cope with uncertainties in wind power generation and load. In the stochastic UC model, the uncertainty of wind power is represented by numerous scenarios of possible wind power output which are often required to ensure the quality of the UC solution. Hence, the limitation with stochastic UC is that the UC problem size and computational requirement increased with the number of scenarios. Another drawback of stochastic $\mathrm{UC}$ is that the scenario generation method usually creates all scenarios based on certain probabilistic distribution assumption without specifying what scenarios can represent the ramp events. However, the probabilistic distribution of the uncertainty parameters is difficult to be acquired in its real world applications.

Robust optimization $[7,8]$ is an alternative uncertainty method to account for the uncertain parameters in optimization problems. Because the bounded uncertainty set considered in robust optimization method with a high solving efficiency is easy to be obtained in reality and the robust optimization strategies can consider the worst case to guarantee the security of power systems operation under all possible scenarios within a given uncertainty set, the robust optimization method applied to UC with uncertain wind power output has become the current research hotspots [914]. A contingency-constrained UC with an $n-K$ security criterion based on robust optimization is proposed in [9], 
where a single-level equivalent robust counterpart of the original problem is formulated. Bertsimas et al. [10] and Jiang et al. [11], respectively, propose a two-stage adaptive robust UC model with adjustable uncertainty budget to reduce the conservativeness of conventional robust optimization. Hu et al. [12] proposes an effective method to acquire robust solutions to the security-constrained UC (SCUC) problem, which takes wind and load uncertainties into account via interval numbers. Based on [11], the uncertainty in demand response is involved in [13] and it assumes that the priceelastic demand curve is also varying within a given range to develop a multistage robust UC model. In [14], a new concept recourse cost defining the upper bound of redispatch cost when uncertainties are revealed is proposed to reduce the conservativeness of robust optimization. All these works [9-14] indicate that robust optimization can ensure system security under the worst-case scenario, and, therefore, it is an effective approach for solving optimization problems with uncertainties.

However, in the existing works [9-14], the robust optimization approaches applied to solve UC problem mainly focus on the adjustment of conservativeness of uncertain parameters including wind power output and load as well as demand response. Wind power spillage which is also called "wind power curtailment" is rarely considered in these robust UC models which have a premise that wind power generation can be completely accommodated no matter how much wind power output and its uncertainty are. As is known, in order to more accurately analyze the impacts of wind power accommodation on on/off schedules and spinning reserve requirements of conventional generators and consider the transmission capacity limits, it is necessary to treat the boundaries of wind power prediction interval as the optimizable decision variables and not merely uncertain parameters in robust UC models to determine the optimal wind power accommodation amount [15].

Therefore, based on a given wind power prediction interval, this paper first proposes a novel double-level robust SCUC problem formulation with optimizable interval of uncertain wind power output. In the proposed model, the boundaries of wind power prediction interval are treated as the optimizable decision variables. And the lower optimization model in the proposed double-level model contains the minimum or maximization formulation which represents the worst-case scenarios for the spinning reserve constraints and the transmission flow constraints. By shrinking the wind power prediction interval, this proposed model can curtail wind power to balance the dispatch cost of conventional generators and dispatch infeasibility penalty cost for wind farms, which is beneficial for mitigating the uncertainty of wind power, especially when the spinning reserve or the capacity of transmission lines is not enough. Furthermore, based on the optimal allowable wind power output interval sent from the operator of dispatch center, the wind farm could make the wind turbines be in the maximum power point tracking operation mode within allowable interval according to the actual wind speed condition to maximize the use of wind energy.
In addition, due to the invalid dual transform in solution process caused by the coupling relation between the discrete and continuous variables, such as the on/off schedules and power output of conventional generators, the proposed model is difficult to be solved and hence this paper proposes a two-stage iterative solution method based on Benders Decomposition (BD). Specifically, the discrete and continuous variables are regarded as the first and the second-stage decision variables, respectively, to eliminate the coupling relation between the discrete and continuous variables, and then the proposed double-level robust interval SCUC problem formulation can be transformed into a single-level and twostage model which can be solved iteratively by BD algorithm.

The remainder of this paper is laid out as follows. First of all, Section 2 describes the double-level robust SCUC model with optimizable interval of uncertain wind power output. Then, the procedure of two-stage iterative solution method based on BD is given in Section 3. In Section 4, the case studies and simulation results analysis are presented. At last, in Section 5, main conclusions are summarized.

\section{Double-Level Robust SCUC Model with Optimizable Interval of Uncertain Wind Power Output}

2.1. Objective Function. The double-level robust interval SCUC model determines the allowable wind power generation interval from system security and economy points of view. The objective function involves two parts: the dispatch cost of conventional generators and the penalty cost of possible wind power spillage over all schedule periods. The dispatch cost contains the fuel cost, start-up and shut-down cost, and spinning reserve cost of generators. The penalty cost of wind power spillage can be expressed to be proportional to the magnitude of the difference between the wind power prediction interval and the allowable wind power output interval to maximize the wind power utilization [16]. Thus, the objective function of proposed UC model is defined as

$$
\begin{aligned}
\min & \left\{\sum_{t=1}^{N T} \sum_{i=1}^{N I}\left(\sum_{k=1}^{N K} c_{k i} p_{k i t}+c_{\min , i} u_{i t}\right)\right. \\
& +\sum_{t=1}^{N T} \sum_{i=1}^{N I}\left(c_{\mathrm{ru}, i} r_{i t}^{\mathrm{u}}+c_{\mathrm{rd}, i} r_{i t}^{\mathrm{d}}\right)+\sum_{t=1}^{N T} \sum_{i=1}^{N I} c_{\mathrm{su}, i} u_{i t}\left(1-u_{i, t-1}\right) \\
& +\sum_{t=1}^{N T} \sum_{i=1}^{N I} c_{\mathrm{sd}, i} u_{i, t-1}\left(1-u_{i t}\right) \\
& \left.+\sum_{t=1}^{N T} \sum_{w=1}^{N W} V_{w}\left(\left(\bar{p}_{w t}^{\mathrm{w}}-\overline{\widehat{p}}_{w t}^{\mathrm{w}}\right)+\left(\underline{p}_{w t}^{\mathrm{w}}-\underline{\underline{p}}_{w t}^{\mathrm{w}}\right)\right)\right\}
\end{aligned}
$$

where NT is the number of time periods in the schedule horizon; the fuel cost of conventional generators is usually expressed as a piecewise linearization of quadratic convex function, NI and NK are the number of conventional generators and the segment number of piecewise linearized function, respectively; $N W$ is the number of wind farms; $c_{k i}$ 
and $c_{\min , i}$ are cost slope of segment $k$ and minimum fuel cost, respectively, of generator $i$, and $c_{k i}$ meets $c_{1 i} \leq c_{2 i} \leq \cdots \leq c_{N K i}$; $p_{k i t}$ is the power output of generator $i$ in segment $k$ during period $t ; u_{i t}$ denoting the on/off schedule of generator $i$ during period $t$ is a binary variable; $r_{i t}^{\mathrm{u}}$ and $r_{i t}^{\mathrm{d}}$ are up- and downspinning reserve amounts of generator $i$, respectively; $c_{\mathrm{ru}, i}$ and $c_{\mathrm{rd}, i}$ are the up- and down-spinning reserve cost coefficients of generator $i$, respectively; $c_{\mathrm{su}_{,}}$and $c_{\mathrm{sd}, i}$ are the start-up and shut-down cost coefficients of generator $i$, respectively; $\bar{p}_{w t}^{\mathrm{w}}$, $\underline{p}_{w t}^{\mathrm{w}}, \widehat{\widehat{p}}_{w t}^{\mathrm{w}}$, and $\underline{\hat{p}}_{w t}^{\mathrm{w}}$ are the upper/lower limits of wind power prediction interval and the upper/lower limits of allowable wind power output interval for wind farm $w$, respectively; $V_{w}$ is the penalty coefficient of the upper/lower limit deviation of wind power output interval for wind farm $w$.

\subsection{Constraints}

\subsubsection{Power Balance Constraint}

$$
\begin{aligned}
\sum_{i=1}^{N I} p_{i t}+\sum_{w=1}^{N W} p_{w t}^{\mathrm{w}} & =\sum_{i=1}^{N I}\left(u_{i t} p_{\min , i}+\sum_{k=1}^{N K} p_{k i t}\right)+\sum_{w=1}^{N W} p_{w t}^{\mathrm{w}} \\
& =\sum_{j=1}^{N J} L_{\mathrm{f}, j t},
\end{aligned}
$$

where NJ is the number of load buses; $L_{\mathrm{f}, j t}$ is the predicted load of bus $j$ during period $t ; p_{i t}$ and $p_{\min , i}$ are the power output during period $t$ and the minimum power output of generator $i$, respectively; $p_{w t}^{\mathrm{w}}$ is the most economic power output for wind farm $w$ during period $t$.

\subsubsection{The Upper and Lower Limits for the Power Output of Conventional Generators}

$$
\begin{gathered}
0 \leq p_{1 i t} \leq u_{i t}\left(p_{\mathrm{g}, 1 i}-p_{\min , i}\right) \\
0 \leq p_{2 i t} \leq u_{i t}\left(p_{\mathrm{g}, 2 i}-p_{\mathrm{g}, 1 i}\right) \\
\vdots \\
0 \leq p_{N K i t} \leq u_{i t}\left(p_{\text {max }, i}-p_{\mathrm{g},(N K-1) i}\right),
\end{gathered}
$$

where $p_{\min , i}, p_{\mathrm{g}, 1 i}, p_{\mathrm{g}, 2 i}, \ldots, p_{\mathrm{g},(N K-1) i}$, and $p_{\max , i}$ are the $N K$ power points, respectively, of linearized power output interval $\left[p_{\min , i}, p_{\max , i}\right]$ of generator $i$.

2.2.3. The Minimum On/Off Schedule Time Constraints of Conventional Generators. $U T_{i}$ is introduced to show the ontime of generator $i$ needed to be maintained at the initial schedule period, and $U T_{i}$ can be calculated as

$$
U T_{i}=\max \left(0, \min \left(N T,\left(T_{\mathrm{min}, i}^{\mathrm{on}}-T_{i 0}^{\mathrm{on}}\right) u_{i 0}\right)\right),
$$

where $T_{\text {min, } i}^{\text {on }}$ is the minimum up-time of generator $i ; T_{i 0}^{\text {on }}$ is the on-time of generator $i$ at the initial schedule period; $u_{i 0}$ denotes the on/off schedule of generator $i$ at the initial schedule period. If the generator needs shut-down, it must meet the minimum up-time as long as it is in on schedule, and hence this constraint can be listed as follows:

$$
\begin{gathered}
u_{i t}=1 \quad\left(t=1,2, \ldots, U T_{i}\right), \\
s_{i t} T_{\min , i}^{\mathrm{on}} \leq \sum_{\tau=t}^{t+T_{\min , i}^{\mathrm{on}}-1} u_{i \tau} \\
\quad\left(t=U T_{i}+1, \ldots, N T-T_{\min , i}^{\mathrm{on}}+1\right), \\
s_{i t}(N T-t+1) \leq \sum_{\tau=t}^{N T} u_{i \tau} \\
\quad\left(t=N T-T_{\min , i}^{\mathrm{on}}+2, \ldots, N T\right),
\end{gathered}
$$

where a binary variable $s_{i t}$ is introduced to judge whether the generator $i$ is in the start-up process during period $t$.

Similarly, $D T_{i}$ is introduced to show the off-time of generator $i$ needed to be maintained at the initial schedule period, and $D T_{i}$ can be calculated as

$$
D T_{i}=\max \left(0, \min \left(N T,\left(T_{\mathrm{min}, i}^{\mathrm{off}}-T_{i 0}^{\mathrm{off}}\right)\left(1-u_{i 0}\right)\right)\right),
$$

where $T_{\mathrm{min}, i}^{\mathrm{off}}$ is the minimum down-time of generator $i ; T_{i 0}^{\mathrm{off}}$ is the off-time of generator $i$ at the initial schedule period. If the generator needs start-up, it must meet the minimum downtime as long as it is in off schedule, and hence this constraint can be listed as follows:

$$
\begin{gathered}
u_{i t}=0 \quad\left(t=1,2, \ldots, D T_{i}\right), \\
\left(1-d_{i t}\right) T_{\mathrm{min}, i}^{\mathrm{off}} \geq \sum_{\tau=t}^{t+T_{\mathrm{min}, i}^{\mathrm{off}}-1} u_{i \tau} \\
\left(t=D T_{i}+1, \ldots, N T-T_{\mathrm{min}, i}^{\mathrm{off}}+1\right),
\end{gathered}
$$

$$
\begin{aligned}
\left(1-d_{i t}\right)(N T-t+1) \geq & \sum_{\tau=t}^{N T} u_{i \tau} \\
& \left(t=N T-T_{\text {min }, i}^{\text {off }}+2, \ldots, N T\right),
\end{aligned}
$$

where a binary variable $d_{i t}$ is introduced to judge whether the generator $i$ is in the shut-down process during period $t . s_{i t}, d_{i t}$, and $u_{i t}$ satisfy

$$
\begin{aligned}
& s_{i t}-d_{i t}=u_{i t}-u_{i, t-1}, \\
& s_{i t}+d_{i t} \leq 1 .
\end{aligned}
$$

In addition, the start-up cost and shut-down cost of generator $i$ in the objective function (1) can be linearized to $c_{\mathrm{su}, i} s_{i t}$ and $c_{\mathrm{sd}, i} d_{i t}$, respectively.

\subsubsection{Spinning Reserve Constraints of Conventional Generators}

$$
\begin{aligned}
& r_{i t}^{\mathrm{u}} \leq \min \left(u_{i t} p_{\max , i}-p_{i t}, u_{i t} r_{i}^{\mathrm{u}} \Delta T\right), \\
& r_{i t}^{\mathrm{d}} \leq \min \left(p_{i t}-u_{i t} p_{\min , i}, u_{i t} r_{i}^{\mathrm{d}} \Delta T\right),
\end{aligned}
$$


where $r_{i}^{\mathrm{u}}$ and $r_{i}^{\mathrm{d}}$ are the ramp-up and ramp-down rates of generator $i$, respectively; $\Delta T$ is the time resolution of per schedule period.

The spinning reserve constraint violation of conventional generator may cause wind power spillage. From the dynamic response ability of systems, wind power output mutation can lead to the decrease of adjustable capacity of generators. When the adjustable capacity of systems is smaller, the security level of systems is lower, and this scenario will be worse. Therefore, the worst-case scenarios for the spinning reserve constraints should be satisfied to guarantee the system security, which can be formulated as (10)-(13):

$$
\begin{aligned}
& R_{t}^{\mathrm{u}}=\min _{p_{w t}^{\mathrm{w}, 1}}\left(\sum_{i=1}^{N I} p_{i t}+\sum_{i=1}^{N I} r_{i t}^{\mathrm{u}}+\sum_{i=1}^{N W} p_{w t}^{\mathrm{w}, 1}-\sum_{j=1}^{N J} L_{\mathrm{f}, j t}\right) \\
& \text { s.t. } \quad \widehat{p}_{w t}^{\mathrm{w}} \leq p_{w t}^{\mathrm{w}, 1} \leq \overline{\widehat{p}}_{w t}^{\mathrm{w}} \quad \forall w \in \mathbf{W}, \\
& R_{t}^{\mathrm{u}} \geq R_{\min , t}^{\mathrm{u}}, \\
& R_{t}^{\mathrm{d}}=\min _{p_{w t}^{\mathrm{w}, 2}}\left(\sum_{j=1}^{N J} L_{\mathrm{f}, j t}-\sum_{i=1}^{N I} p_{i t}+\sum_{i=1}^{N I} r_{i t}^{\mathrm{d}}-\sum_{i=1}^{N W} p_{w t}^{\mathrm{w}, 2}\right) \\
& \text { s.t. } \quad \widehat{p}_{w t}^{\mathrm{w}} \leq p_{w t}^{\mathrm{w}, 2} \leq \overline{\widehat{p}}_{w t}^{\mathrm{w}} \quad \forall w \in \mathbf{W}, \\
& R_{t}^{\mathrm{d}} \geq R_{\min , t}^{\mathrm{d}},
\end{aligned}
$$

where $\mathbf{W}$ is the set of wind farms; $R_{t}^{\mathrm{u}}$ and $R_{t}^{\mathrm{d}}$ are upand down-spinning reserve, respectively, supplied by systems under the worst-case scenario during period $t ; p_{w t}^{\mathrm{w}, 1}$ and $p_{w t}^{\mathrm{w}, 2}$ are the power output of wind farm $w$ under the worst-case scenario for the up- and down-spinning reserve constraints, respectively, during period $t ; R_{\min , t}^{\mathrm{u}}$ and $R_{\min , t}^{\mathrm{d}}$ are the minimum up- and down-spinning reserve requirements of systems, respectively, during period $t$.

2.2.5. Ramp-Rate Limits for Conventional Generators. From the dynamic response ability of systems, the ramp-rate limits for conventional generators under the worst-case scenario can be formulated as follows:

$$
\begin{aligned}
& \sum_{i=1}^{N I} \Delta r_{i t}^{\mathrm{u}}=\sum_{i=1}^{N I} r_{i t}^{\mathrm{u}}-R_{t}^{\mathrm{u}}, \\
& \sum_{i=1}^{N I} \Delta r_{i t}^{\mathrm{d}}=\sum_{i=1}^{N I} r_{i t}^{\mathrm{d}}-R_{t}^{\mathrm{d}}, \\
& 0 \leq \Delta r_{i t}^{\mathrm{u}} \leq r_{i t}^{\mathrm{u}}, \\
& 0 \leq \Delta r_{i t}^{\mathrm{d}} \leq r_{i t}^{\mathrm{d}}, \\
& p_{i t}+\Delta r_{i t}^{\mathrm{u}}-p_{i, t-1}+\Delta r_{i, t-1}^{\mathrm{d}} \leq\left(1-s_{i t}\right) r_{i}^{\mathrm{u}} \Delta T+s_{i t} p_{\min , i}, \\
& p_{i, t-1}+\Delta r_{i, t-1}^{\mathrm{u}}-p_{i t}+\Delta r_{i t}^{\mathrm{d}} \\
& \quad \leq\left(1-d_{i t}\right) r_{i}^{\mathrm{d}} \Delta T+d_{i t} p_{\min , i},
\end{aligned}
$$

where $\Delta r_{i t}^{\mathrm{u}}$ and $\Delta r_{i t}^{\mathrm{d}}$ are up and down power generation adjusting amounts for generator $i$ under the worst-case scenarios (10) and (12), respectively, during period $t$. The generators may be in off schedule in UC during periods $t-1$ and $t$; hence it assumes in (18) and (19) that the power output of generator will reach the minimum value once the generator starts up and the power output of generator is required to be the minimum value before it shuts down.

2.2.6. Transmission Flow Constraints. The transmission flow constraints violation may also lead to wind power spillage. From the network security, the wind power output boundary value will result in the maximum load rate of transmission line achieved. When the load rate of transmission line is higher, the security level of systems is lower, and this scenario will be worse. Therefore, the worst-case scenarios for the transmission flow constraints should be satisfied to guarantee the network security, which can be formulated as follows:

$$
\begin{aligned}
F_{l t}^{+}=\max _{p_{w t}^{\mathrm{w}, 3}} & \left(\sum_{b=1}^{N B} S F_{l b}\left(\sum_{i \wedge b} p_{i t}+\sum_{w \wedge b} p_{w t}^{\mathrm{w}, 3}-\sum_{j \wedge b} L_{\mathrm{f}, j t}\right)\right) \\
& \leq T L_{l} \\
\text { s.t. } \quad \widehat{p}_{w t}^{\mathrm{w}} \leq p_{w t}^{\mathrm{w}, 3} \leq \overline{\widehat{p}}_{w t}^{\mathrm{w}} & \quad \forall w \in \mathbf{W}, l=1,2, \ldots, N L, \\
F_{l t}^{-}=\min _{p_{w t}^{\mathrm{w}, 4}} & \left(\sum_{b=1}^{N B} S F_{l b}\left(\sum_{i \wedge b} p_{i t}+\sum_{w \wedge b} p_{w t}^{\mathrm{w}, 4}-\sum_{j \wedge b} L_{\mathrm{f}, j t}\right)\right) \\
& \geq-T L_{l} \\
\text { s.t. } \quad & \underline{\hat{p}}_{w t}^{\mathrm{w}} \leq p_{w t}^{\mathrm{w}, 4} \leq \overline{\widehat{p}}_{w t}^{\mathrm{w}}
\end{aligned}
$$$$
\forall w \in \mathbf{W}, l=1,2, \ldots, N L
$$

where $N B$ is the number of buses; $N L$ is the number of transmission lines; $S F_{l b}$ referring to [17] is power transfer distribution factor of bus $b$ to line $l ; i \wedge b, w \wedge b$, and $j \wedge b$ represent the generator $i$, wind farm $w$, and load $j$ connected to bus $b$, respectively; $F_{l t}^{+}$and $F_{l t}^{-}$are the maximum positive and negative power flows of line $l$ during period $t ; p_{w t}^{w, 3}$ and $p_{w t}^{w, 4}$ are the decision variables of power output of wind farm under the worst-case scenario for the positive and negative transmission flow constraint; $T L_{l}$ is the capacity limit of line $l$.

\subsubsection{Allowable Wind Power Output Interval Constraints}

$$
\begin{aligned}
& \underline{\hat{p}}_{w t}^{\mathrm{w}} \leq p_{w t}^{\mathrm{w}} \leq \overline{\widehat{p}}_{w t}^{\mathrm{w}} \leq \bar{p}_{w t}^{\mathrm{w}}, \\
& \underline{\widehat{p}}_{w t}^{\mathrm{w}} \leq \underline{p}_{w t}^{\mathrm{w}} .
\end{aligned}
$$

The upper limits of the maximum allowable wind power output interval must be lower than the upper limits of wind power prediction interval for every wind farm. Meantime, the lower limits of maximum allowable wind power output 
interval must be smaller than or equal to the lower limits of wind power prediction interval for practical generation schedule.

\section{Two-Stage Iterative Solution Method Based on BD Algorithm}

Because the lower optimization models (10), (12), (20), and (21) contain the minimum or maximization formulation, the double-level robust interval SCUC model described in Section 2 cannot be solved efficiently. By the equivalent transformation of the minimum and maximization expressions based on linear duality theory [18], the double-level model needs to be transformed into a single-level model to be solved. However, due to the coupling relation between the continuous variables and the discrete variables of generator schedule in the upper and lower models, the upper model requirements for the lower model are not merely the maximum and minimum power generation capacity constraints. Therefore, the original duality transformation of the two-level model to a single-level model applied to economic dispatch will be invalid in the UC, and the corresponding double-level interval SCUC model is difficult to be solved.

For this reason, in this section, firstly, the discrete variables are regarded as the first-stage decision variables, and the continuous variables are regarded as the second-stage decision variables to eliminate the decouple relationship between the discrete and continuous variables. Then according to the linear duality theory, the double-level robust interval SCUC model described in Section 2 can be transformed into an equivalent single-level and two-stage robust interval SCUC model. Finally, the two-stage SCUC model can be solved iteratively by $\mathrm{BD}$ algorithm.

3.1. Construction of a Single-Level and Two-Stage Robust Interval SCUC Model. In order to facilitate the description of the establishment process of the two-stage robust interval SCUC model and the solution method based on $\mathrm{BD}$ algorithm, firstly, the double-level model in a compact form is described as follows:

$$
\begin{array}{ll}
\min _{\mathbf{x}, \mathbf{y}} & \left(\mathbf{c}^{\mathrm{T}} \mathbf{x}+\mathbf{b}^{\mathrm{T}} \mathbf{y}\right) \\
\text { s.t. } & \mathbf{F x} \leq \mathbf{f}, \mathbf{E x}=\mathbf{e} \\
& \mathbf{H y} \leq \mathbf{h}, \mathbf{A x}+\mathbf{B y} \leq \mathbf{m}, \mathbf{I x}+\mathbf{J y}=\mathbf{n}
\end{array}
$$

where (23) is the objective function of the upper model, where $\mathbf{x}$ is the column vector of decision variables composed of the discrete variables $u_{i t}, s_{i t}$, and $d_{i t}$, $\mathbf{y}$ is the column vector of decision variables composed of the continuous variables $p_{k i t}$, $\overline{\bar{p}}_{w t}^{\mathrm{w}}, \underline{p}_{w t}^{\mathrm{w}}, p_{w t}^{\mathrm{w}}, r_{i t}^{\mathrm{u}}, r_{i t}^{\mathrm{d}}, \Delta r_{i t}^{\mathrm{u}}, \Delta r_{i t}^{\mathrm{d}}, p_{w t}^{\mathrm{w}, 1}, p_{w t}^{\mathrm{w}, 2}, p_{w t}^{\mathrm{w}, 3}$, and $p_{w t}^{\mathrm{w}, 4}$, the coefficient column vectors $\mathbf{c}$ and $\mathbf{b}$ are acquired based on (1); the constraint conditions $\mathbf{F x} \leq \mathbf{f}$ and $\mathbf{E x}=\mathbf{e}$ of the upper model represent (5) and (7)-(8), Hy $\leq \mathbf{h}$ represents (16), (17), and (22), Ax $+\mathbf{B y} \leq \mathbf{m}$ represents (3), (9), (18), and (19), $\mathbf{I x}+\mathbf{J y}=\mathbf{n}$ represents (2); the extremal problem $\min _{\mathbf{y}}(\mathbf{C x}+\mathbf{D y})=\mathbf{K y}$ of the lower model represents (10), (12), (14), and (15), $\min _{\mathbf{y}}(\mathbf{M x}+\mathbf{N y}) \geq \mathbf{v}$ represents (10)-(13), (20), and (21), F, E, H, A, B, I, J, C, D, K, G, M, N, and $\mathbf{Q}$ are the coefficient matrixes, and $\mathbf{f}, \mathbf{e}, \mathbf{h}, \mathbf{m}, \mathbf{n}, \mathbf{v}, \mathbf{g}$, and $\mathbf{q}$ are the coefficient column vectors.

The specific construction process of the single-level and two-stage robust interval SCUC model can be described as follows.

Step 1. The discrete variable vector $\mathbf{x}$ is regarded as the firststage decision variable, and the continuous variable vector $\mathbf{y}$ is regarded as the second-stage decision variable. Then the double-level models (23) and (24) can be equivalent to the double-level and two-stage models (25)-(26)

$$
\begin{array}{ll}
\min _{\mathbf{x}} & \left(\mathbf{c}^{\mathrm{T}} \mathbf{x}+\min _{\mathbf{y} \in \Omega(\mathbf{x})} \mathbf{b}^{\mathrm{T}} \mathbf{y}\right) \\
\text { s.t. } & \mathbf{F x} \leq \mathbf{f}, \mathbf{E} \mathbf{x}=\mathbf{e}, \mathbf{x} \in\{0,1\},
\end{array}
$$

where

$$
\Omega(\mathbf{x})= \begin{cases}\mathbf{y}: \mathbf{H y} \leq \mathbf{h}, \mathbf{A x}+\mathbf{B y} \leq \mathbf{m}, \mathbf{I x}+\mathbf{J y}=\mathbf{n}, & \mathbf{y} \geq 0 \\
\begin{cases}\min _{\mathbf{y}}(\mathbf{C} \mathbf{x}+\mathbf{D y})=\mathbf{K y} \\
\text { s.t. } \quad \mathbf{G y} \leq \mathbf{g},\end{cases} & \left\{\begin{array}{l}
\min _{\mathbf{y}} \quad(\mathbf{M x}+\mathbf{N y}) \geq \mathbf{v} \\
\text { s.t. }
\end{array}\right.\end{cases}
$$

Step 2. In the second-stage model $\min _{\mathbf{y} \in \Omega(\mathbf{x})} \mathbf{b}^{\mathrm{T}} \mathbf{y}, \mathbf{x}$ is a given vector, and $\min _{\mathbf{y} \in \Omega(\mathbf{x})} \mathbf{b}^{\mathrm{T}} \mathbf{y}$ can be regarded as a double-level robust interval economic dispatch model which cannot be solved directly due to the coupling relationship between the upper and lower model. However, according to a linear duality theory, from the goal of minimizing $\mathbf{b}^{\mathrm{T}} \mathbf{y}$ in the upper 
model, the constrained extremal problem in the lower model $\Omega(\mathbf{x})$ can be equivalent to the duality problem of the lower model. Hence, the double-level optimization model can be transformed to a single-level model to be solved.

In the constraint conditions of the lower models (10), (12), (20), and (21), the left-hand and right-hand constraints of $p_{w t}^{\mathrm{w}, 1}, p_{w t}^{\mathrm{w}, 2}, p_{w t}^{\mathrm{w}, 3}$, and $p_{w t}^{\mathrm{w}, 4}$ are all decision variables, and the direct duality transformation may cause a nonlinear term of the product of two decision variables. Hence, these constraints need firstly simplification treatment. Take $p_{w t}^{\mathrm{w}, 1}$, for example, introduce a new continuous variable $\lambda_{w t}^{1}(0 \leq$ $\left.\lambda_{w t}^{1} \leq 1\right)$, and let $p_{w t}^{\mathrm{w}, 1}=\underline{\hat{p}}_{w t}^{\mathrm{w}}+\lambda_{w t}^{1}\left(\overline{\widehat{p}}_{w t}^{\mathrm{w}}-\underline{\hat{p}}_{w t}^{\mathrm{w}}\right)$. Accordingly, $p_{w t}^{\mathrm{w}, 2}, p_{w t}^{\mathrm{w}, 3}$, and $p_{w t}^{\mathrm{w}, 4}$ are substituted by $\lambda_{w t}^{2}, \lambda_{w t}^{3}$, and $\lambda_{w t}^{4}$, respectively.

The lower optimization models (10), (12), (20), and (21) are, respectively, substituted by the corresponding duality problem to ensure that the dual objective function of the lower optimization model is the upper or lower bound of the original optimization model. Before the duality transformation, the decision variables of the lower optimization model are $\lambda_{w t}^{1}, \lambda_{w t}^{2}, \lambda_{w t}^{3}$ and $\lambda_{w t}^{4}$. Correspondingly, after the duality transformation the duality variables are $\alpha_{w t}, \beta_{w t}, \delta_{w t}$, and $\varphi_{w t}$, respectively, and the duality model can be formulated as

$$
\begin{array}{r}
\sum_{i=1}^{N I} p_{i t}+\sum_{i=1}^{N I} r_{i t}^{\mathrm{u}}+\sum_{w=1}^{N W} \underline{\widehat{p}}_{w t}^{\mathrm{w}}-\sum_{w=1}^{N W} \alpha_{w t}-\sum_{j=1}^{N J} L_{\mathrm{f}, j t} \geq R_{\min , t}^{\mathrm{u}} \\
\alpha_{w t} \geq-\left(\overline{\widehat{p}}_{w t}^{\mathrm{w}}-\underline{\hat{p}}_{w t}^{\mathrm{w}}\right), \forall w \in \mathbf{W},
\end{array}
$$

$$
\begin{array}{r}
\sum_{j=1}^{N J} L_{\mathrm{f}, j t}-\sum_{i=1}^{N I} p_{i t}+\sum_{i=1}^{N I} r_{i t}^{\mathrm{d}}-\sum_{w=1}^{N W} \underline{\hat{p}}_{w t}^{\mathrm{w}}-\sum_{w=1}^{N W} \beta_{w t} \geq R_{\mathrm{min}, t}^{\mathrm{d}} \\
\beta_{w t} \geq \overline{\widehat{p}}_{w t}^{\mathrm{W}}-\underline{\hat{p}}_{w t}^{\mathrm{w}}, \forall w \in \mathbf{W},
\end{array}
$$

$$
\begin{array}{r}
\sum_{b=1}^{N B} S F_{l b}\left(\sum_{i \wedge b} p_{i t}+\sum_{w \wedge b} \underline{\hat{p}}_{w t}^{\mathrm{w}}+\sum_{w \wedge b} \delta_{w t}-\sum_{j \wedge b} L_{\mathrm{f}, j t}\right) \leq U_{l} \\
\delta_{w t} \geq \widehat{\widehat{p}}_{w t}^{\mathrm{w}}-\underline{\widehat{p}}_{w t}^{\mathrm{w}}, \forall w \in \mathbf{W},
\end{array}
$$

$$
\begin{array}{r}
\sum_{b=1}^{N B} S F_{l b}\left(\sum_{i \wedge b} p_{i t}+\sum_{w \wedge b} \underline{\hat{p}}_{w t}^{\mathrm{w}}-\sum_{w \wedge b} \varphi_{w t}-\sum_{j \wedge b} L_{\mathrm{f}, j t}\right) \geq-U_{l} \\
\varphi_{w t} \geq-\left(\overline{\widehat{p}}_{w t}^{\mathrm{w}}-\underline{\hat{p}}_{w t}^{\mathrm{w}}\right), \forall w \in \mathbf{W} .
\end{array}
$$

The general form of this transformation based on linear duality theory is given in Appendix.

Step 3. $R_{t}^{\mathrm{u}}$ and $R_{t}^{\mathrm{d}}$ in (14) and (15) are substituted by the left side term in (27) and (28), respectively. Then the secondstage and double-level optimization model $\min _{\mathbf{y} \in \Omega(\mathbf{x})} \mathbf{b}^{\mathrm{T}} \mathbf{y}$ can be transformed to a single-level model $\min _{\mathbf{z} \in \Omega^{\prime}(\mathbf{x})} \mathbf{b}^{\mathrm{T}} \mathbf{z}$, where $\Omega^{\prime}(\mathbf{x})$ satisfies

$$
\begin{aligned}
& \Omega^{\prime}(\mathbf{x}) \\
& \quad=\{\mathbf{z}: \mathbf{L z} \leq \mathbf{1}, \mathbf{P x}+\mathbf{S z} \leq \mathbf{p}, \mathbf{W} \mathbf{x}+\mathbf{V z}=\mathbf{w}, \mathbf{z} \geq \mathbf{0}\},
\end{aligned}
$$

where $\mathbf{z}$ is the column vector of decision variables composed of the continuous variables $p_{k i t}, \overline{\widehat{p}}_{w t}^{\mathrm{w}}, \underline{\hat{p}}_{w t}^{\mathrm{w}}, p_{w t}^{\mathrm{w}}, r_{i t}^{\mathrm{u}}, r_{i t}^{\mathrm{d}}, \Delta r_{i t}^{\mathrm{u}}, \Delta r_{i t}^{\mathrm{d}}$, $\alpha_{w t}, \beta_{w t}, \delta_{w t}$, and $\varphi_{w t} ; \mathbf{L z} \leq \mathbf{1}$ denotes the second constraints of (16), (17), (22), and (27)-(30); $\mathbf{P x}+\mathbf{S z} \leq \mathbf{p}$ denotes the first constraints of (3), (9), (18), (19), and (27)-(30); $\mathbf{W} \mathbf{x}+\mathbf{V z}=\mathbf{w}$ denotes (2), (14), and (15); $\mathbf{L}, \mathbf{P}, \mathbf{S}, \mathbf{W}$, and $\mathbf{V}$ are coefficient matrixes; $\mathbf{l}, \mathbf{p}$, and $\mathbf{w}$ are coefficient column vectors.

Finally the single-level and two-stage robust interval SCUC model equivalent to the original double-level models (23) and (24) can be obtained:

$$
\begin{aligned}
& \min _{\mathbf{x}}\left(\mathbf{c}^{\mathrm{T}} \mathbf{x}+\min _{\mathbf{z} \in \Omega^{\prime}(\mathbf{x})} \mathbf{b}^{\mathrm{T}} \mathbf{z}\right) \\
& \text { s.t. } \quad \mathbf{F x} \leq \mathbf{f}, \mathbf{E x}=\mathbf{e}, \mathbf{x} \in\{0,1\} .
\end{aligned}
$$

3.2. Procedure of the Two-Stage Iterative Solution Method Based on BD Algorithm. The duality subproblem of the second-stage optimal subproblem $\min _{\mathbf{z} \in \Omega^{\prime}(\mathbf{x})} \mathbf{b}^{\mathrm{T}} \mathbf{z}$ in the twostage robust interval SCUC model (32) is

$$
\begin{array}{ll}
\max _{\boldsymbol{\omega}, \boldsymbol{\eta}, \boldsymbol{\chi}} & \left((\mathbf{P x}-\mathbf{p})^{\mathrm{T}} \boldsymbol{\omega}-\mathbf{l}^{\mathrm{T}} \boldsymbol{\eta}+(\mathbf{w}-\mathbf{W} \mathbf{x})^{\mathrm{T}} \boldsymbol{\chi}\right) \\
\text { s.t. } & -\mathbf{S}^{\mathrm{T}} \boldsymbol{\omega}-\mathbf{L}^{\mathrm{T}} \boldsymbol{\eta}+\mathbf{V}^{\mathrm{T}} \boldsymbol{\chi} \leq \mathbf{b} \\
& \boldsymbol{\omega} \geq 0, \boldsymbol{\eta} \geq 0, \boldsymbol{\chi} \text { is unconstrained, }
\end{array}
$$

where the variables $\boldsymbol{\omega}, \boldsymbol{\eta}$, and $\boldsymbol{\chi}$ are the duality variables of the variable $\mathbf{z}$. The feasible solution set (34) of the duality subproblem is independent of the discrete variable $\mathbf{x}$, and, based on the duality theory, the two-stage robust interval SCUC model can be expressed as

$$
\begin{array}{ll}
\min _{\mathbf{x}} & \left(\mathbf{c}^{\mathrm{T}} \mathbf{x}+\max _{\boldsymbol{\omega}, \boldsymbol{\eta}, \boldsymbol{\chi}}\left((\mathbf{P} \mathbf{x}-\mathbf{p})^{\mathrm{T}} \boldsymbol{\omega}-\mathbf{1}^{\mathrm{T}} \boldsymbol{\eta}+(\mathbf{w}-\mathbf{W} \mathbf{x})^{\mathrm{T}} \boldsymbol{\chi}\right)\right) \\
\text { s.t. } & \mathbf{F x} \leq \mathbf{f}, \mathbf{E} \mathbf{x}=\mathbf{e}, \mathbf{x} \in\{0,1\} .
\end{array}
$$

The pole set and the polar direction set of the feasible solution set (34) for the Benders subproblem are $\mathbf{H}^{\mathrm{P}}$ and $\mathbf{H}^{\mathrm{R}}$, respectively. Introduce the relaxed variable $\theta$, and construct the Benders main problem of the two-stage robust interval SCUC model (35) which can be described as follows:

$$
\begin{array}{ll}
\min _{\mathbf{x}, \theta} & \left(\mathbf{c}^{\mathrm{T}} \mathbf{x}+\theta\right) \\
\text { s.t. } & \mathbf{F x} \leq \mathbf{f}, \mathbf{E x}=\mathbf{e}, \mathbf{x} \in\{0,1\} \\
& \boldsymbol{\omega}_{p}^{\mathrm{T}}(\mathbf{P} \mathbf{x}-\mathbf{p})-\boldsymbol{\eta}_{p}^{\mathrm{T}} \mathbf{1}+\boldsymbol{\chi}_{p}^{\mathrm{T}}(\mathbf{w}-\mathbf{W} \mathbf{x}) \leq \theta \\
& \overline{\boldsymbol{\omega}}_{r}^{\mathrm{T}}(\mathbf{P} \mathbf{x}-\mathbf{p})-\overline{\boldsymbol{\eta}}_{r}^{\mathrm{T}} \mathbf{1}+\overline{\boldsymbol{\chi}}_{r}^{\mathrm{T}}(\mathbf{w}-\mathbf{W} \mathbf{x}) \leq 0 \\
& \forall\left(\boldsymbol{\omega}_{p}, \boldsymbol{\eta}_{p}, \boldsymbol{\chi}_{p}\right) \in \mathbf{H}^{\mathrm{P}}, \forall\left(\overline{\boldsymbol{\omega}}_{r}, \overline{\boldsymbol{\eta}}_{r}, \overline{\boldsymbol{\chi}}_{r}\right) \in \mathbf{H}^{\mathrm{R}},
\end{array}
$$




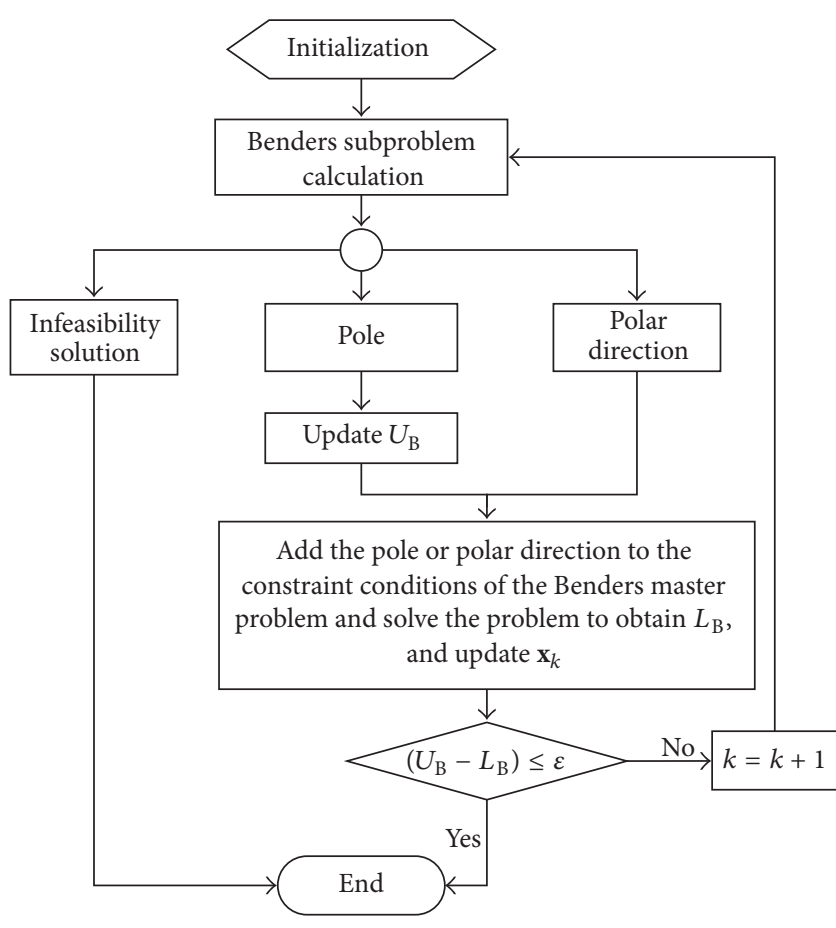

FIGURE 1: Flow chart of the solution method based on BD.

where (38) and (39) are the Benders optimality and feasibility cutting planes, respectively, connecting the master problem and subproblem.

Referring to $[19,20]$, the flow chart of the solution method based on BD is shown in Figure 1. Initialization firstly: set the iteration count $k=1$, the given initial upper bound $U_{\mathrm{B}}$ and lower bound $L_{\mathrm{B}}$, the convergence tolerance $\varepsilon>0, \mathbf{H}^{\mathrm{P}}=$ $\emptyset$, and $\mathbf{H}^{\mathrm{R}}=\emptyset$; construct a feasible solution $\mathbf{x}_{0}$. The detailed procedure of the solution method based on $\mathrm{BD}$ can be listed as follows.

Step 1. $\mathbf{x}_{k-1}$ is added to the Benders subproblem (33) and (34) which is a linear programming problem, and the results can be divided into the following three situations.

Case 1. If the subproblem has infeasibility solution, the algorithm terminates.

Case 2. If the subproblem has an unbounded optimal value, it can obtain a polar direction $\left(\overline{\boldsymbol{\omega}}_{r}, \overline{\boldsymbol{\eta}}_{r}, \overline{\boldsymbol{\chi}}_{r}\right)$ and continues the next step.

Case 3. If the subproblem has an optimal value, it can get a pole $\left(\boldsymbol{\omega}_{p}, \boldsymbol{\eta}_{p}, \boldsymbol{\chi}_{p}\right)$. Use this value to update the upper bound $U_{\mathrm{B}}=\mathbf{c}^{\mathrm{T}} \mathbf{x}_{k-1}+\left(\mathbf{P} \mathbf{x}_{k-1}-\mathbf{p}\right)^{\mathrm{T}} \boldsymbol{\omega}_{p}-\mathbf{1}^{\mathrm{T}} \boldsymbol{\eta}_{p}+\left(\mathbf{w}-\mathbf{W} \mathbf{x}_{k-1}\right)^{\mathrm{T}} \boldsymbol{\chi}_{p}$ and execute the next step.

Step 2. The pole or polar direction obtained in Step 1 is added to the constraint conditions $\mathbf{H}^{\mathrm{P}}$ and $\mathbf{H}^{\mathrm{R}}$ of the benders master problem. The relaxed benders master problem (36)-(40) is a mixed integer linear programming problem, and, by CPLEX solver, the optimal solution $\left(\mathbf{x}_{k}, \theta_{k}\right)$ and the lower bound $L_{\mathrm{B}}=$ $\mathbf{c}^{\mathrm{T}} \mathbf{x}_{k}+\theta_{k}$ are calculated.

Step 3. Check whether $\left(U_{\mathrm{B}}-L_{\mathrm{B}}\right) \leq \varepsilon$ is satisfied or not. If it is satisfied, terminate the loop, return the result $\mathbf{x}_{k}$, and add $\mathbf{x}_{k}$ to $\min _{\mathbf{z} \in \Omega^{\prime}(\mathbf{x})} \mathbf{b}^{\mathrm{T}} \mathbf{z}$ to obtain $\mathbf{z}_{k}$. Otherwise, let $k=k+1$, and go to Step 1 .

In addition, [21] proposes a combination of Benders cutting planes to substitute (39); that is, when the Benders subproblem has infeasibility solution, the Benders master problem adds the following integer cutting plane:

$$
\sum_{x_{r, j}=0} x_{k, j}+\sum_{x_{r, j}=1}\left(1-x_{k, j}\right) \geq 1 .
$$

The purpose of (41) is to obtain the solution of the Benders subproblem by changing the value $\mathbf{x}_{k}$.

\section{Case Studies and Simulation Results Analysis}

4.1. Modified IEEE 26-Generator Reliability Test System. A modified IEEE 26-generator reliability test system is adopted in this paper to verify the effectiveness of the proposed model. In this system, there are 26 thermal generators with a total capacity of $3105 \mathrm{MW}$. The capacity limits of transmission lines, the ramp rates, cost coefficients, and minimum up- and down-time of generators are obtained from [22]. One wind farm is added at bus 14 . Its capacity is set at $600 \mathrm{MW}$. To illustrate the effect of wind power spillage on the determination of SR requirements, Table 1 lists the forecast load and predicted power output interval of wind farm used for testing. It can be observed from the table that the wind power output and load are negatively correlated. In addition, during periods 7 8, the capacity limits of transmission lines between bus 14 and bus 11 and bus 14 and bus 16 are changed to $100 \mathrm{MW}$ from $500 \mathrm{MW}$. The unit penalty cost of wind power spillage $V_{w}=10 \$ /(\mathrm{MW} \cdot \mathrm{h})$. The minimum up- and down-spinning reserve requirements are set at $400 \mathrm{MW}$.

The initialization of each parameter is described as follows: determine the feasible solution $\mathbf{x}_{0}$ considering that each generator is operational during each period; upper bound $U_{\mathrm{B}}=+\infty$ and lower bound $L_{\mathrm{B}}=-\infty$; convergence tolerance $\varepsilon=0.005$.

4.2. Effectiveness Analysis of Solution Strategy Based on BD. The mixed integer linear programming model of the proposed master problem and linear programming model of the proposed subproblem is solved using a commercial solver CPLEX 12.5 under MATLAB environment. The solution result of the subproblem can be checked according to the exit flag value of the solution function in MATLAB. When the duality gap tolerance for CPLEX solver is set at $0.01 \%$, the computation time is about $8.6 \mathrm{~s}$ on a $2.4-\mathrm{GHz}$ Windowsbased workstation with 4 GB of RAM.

To illustrate the effectiveness of the proposed two-stage iterative solution method based on $\mathrm{BD}$, the results of the test system are given in Table 2. From the variation of the upper 
TABLE 1: Forecast data of load and power output interval of wind farm.

\begin{tabular}{lcc}
\hline Hour & Load/MW & Power output interval of wind farm/p.u. \\
\hline 1 & 1700 & {$[0.656,1.000]$} \\
2 & 1730 & {$[0.722,1.000]$} \\
3 & 1690 & {$[0.718,1.000]$} \\
4 & 1700 & {$[0.662,1.000]$} \\
5 & 1750 & {$[0.636,1.000]$} \\
6 & 1850 & {$[0.552,0.878]$} \\
7 & 2000 & {$[0.378,0.618]$} \\
8 & 2430 & {$[0.319,0.529]$} \\
9 & 2540 & {$[0.250,0.426]$} \\
10 & 2600 & {$[0.276,0.464]$} \\
11 & 2670 & {$[0.134,0.252]$} \\
12 & 2590 & {$[0.289,0.483]$} \\
13 & 2390 & {$[0.537,0.736]$} \\
14 & 2050 & {$[0.628,0.992]$} \\
15 & 1820 & {$[0.745,1.000]$} \\
16 & 1750 & {$[0.696,1.000]$} \\
17 & 1700 & {$[0.534,0.852]$} \\
18 & 1730 & {$[0.446,0.720]$} \\
19 & 1860 & {$[0.354,0.582]$} \\
20 & 2150 & {$[0.293,0.489]$} \\
21 & 2400 & {$[0.201,0.351]$} \\
22 & 2480 & {$[0.430,0.694]$} \\
23 & 2200 & {$[0.582,0.922]$} \\
24 & 1840 &
\end{tabular}

TABLE 2: Iterative computation results of test system in each step.

\begin{tabular}{lcc}
\hline$k$ & $U_{\mathrm{B}} / \$$ & $L_{\mathrm{B}} / \$$ \\
\hline 1 & 910483.30 & 550638.41 \\
2 & 910483.30 & 615400.13 \\
3 & 796254.87 & 676003.85 \\
4 & 734035.61 & 734035.61 \\
\hline
\end{tabular}

and lower bounds in this table, it can be observed that the total iteration number is 4 , and the other iterations obtain the poles besides the polar direction obtained in the second iteration. In the iterative process, the upper and lower bounds of the objective function value (1) of the original complex problems are constantly revised; that is, the upper bound is decreasing, the lower bound is increasing, and the optimal value of the original problem is finally approached.

\subsection{Comparison of Proposed Model and Traditional Robust} UC Model. Compared with the traditional robust UC model, the upper and lower bounds of wind power prediction interval in each period are regarded as decision variables to consider the wind power spillage in the proposed model. It can accurately analyze the impacts of wind power accommodation on on/off schedules and spinning reserve requirements of conventional generators and consider the network security limits from the safety and economy of systems operation.

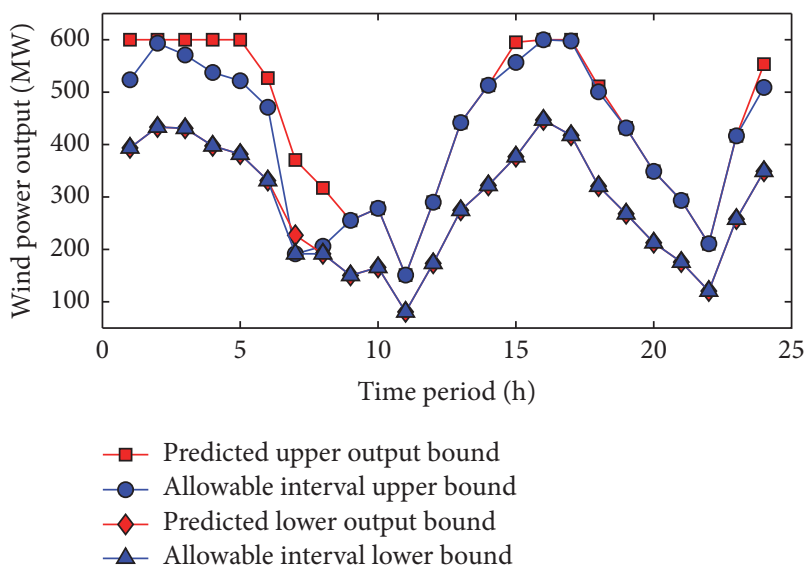

FIGURE 2: Comparison of predicted and optimization allowable upper and lower bound of wind power output interval.

TABLE 3: Comparison of different costs of between the proposed model and conventional robust UC model.

\begin{tabular}{lcc}
\hline Cost & Proposed model & $\begin{array}{c}\text { Conventional robust } \\
\text { UC model }\end{array}$ \\
\hline $\begin{array}{l}\text { Fuel cost/\$ } \\
\text { Start-up and shut-down } \\
\text { costs } \$\end{array}$ & 550796.99 & 552273.81 \\
$\begin{array}{l}\text { Spinning reserve cost/\$ } \\
\text { Penalty cost of wind power } \\
\text { spillage/ }\end{array}$ & 162317.41 & 7951.00 \\
Total cost $/ \$$ & 4040.80 & 165697.75 \\
\hline
\end{tabular}

4.3.1. Analysis of the Necessity of Considering the Wind Power Spillage. From the transmission flow constraints of systems, wind power spillage needs to be considered. The comparison of predicted and optimized allowable interval upper and lower bounds of wind power output is illustrated in Figure 2. From this figure, it can be observed that, during periods 7 and 8 , the capacity limits of transmission lines between buses 14 and 11 and buses 14 and 16 are all $100 \mathrm{MW}$, and the insufficient transmission line capacity makes the allowable wind power output interval less than the predicted wind power output interval in the proposed model. Meanwhile, the traditional robust UC model cannot satisfy the transmission flow constraints and it has no solution.

4.3.2. Analysis of the Impacts of Wind Power Spillage on the Economy Improvement. The capacity limits of transmission lines between buses 14 and 11 and buses 14 and 16 are altered to $500 \mathrm{MW}$ to eliminate the influence of transmission flow constraints. Table 3 compares different costs (fuel cost, startup and shut-down cost, spinning reserve cost, penalty cost of wind power spillage, and total cost) between the proposed model and the conventional robust UC model. Figures 3, 4, and 5 compare the upper and lower bounds of the wind power output interval, the operational schedules of conventional generators, and the up- and down-spinning reserve between the two models, respectively. 


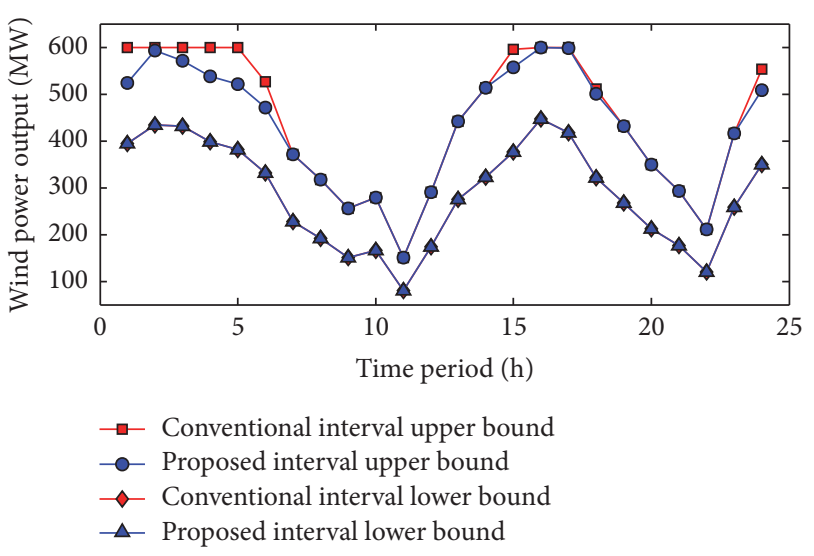

FIGURE 3: Comparison of interval upper and lower bound of wind power output between the proposed model and conventional robust UC model.

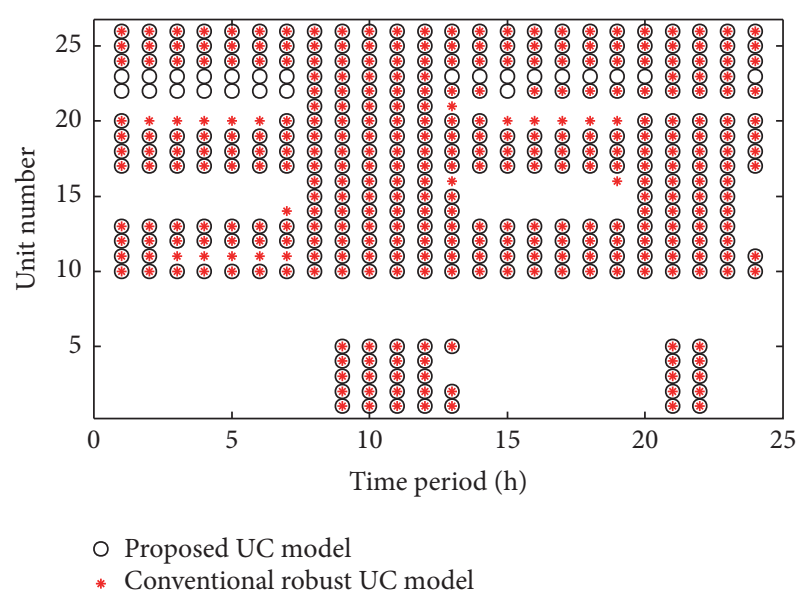

FIgURE 4: Comparison of operational schedules of conventional generators between the proposed model and conventional robust UC model.
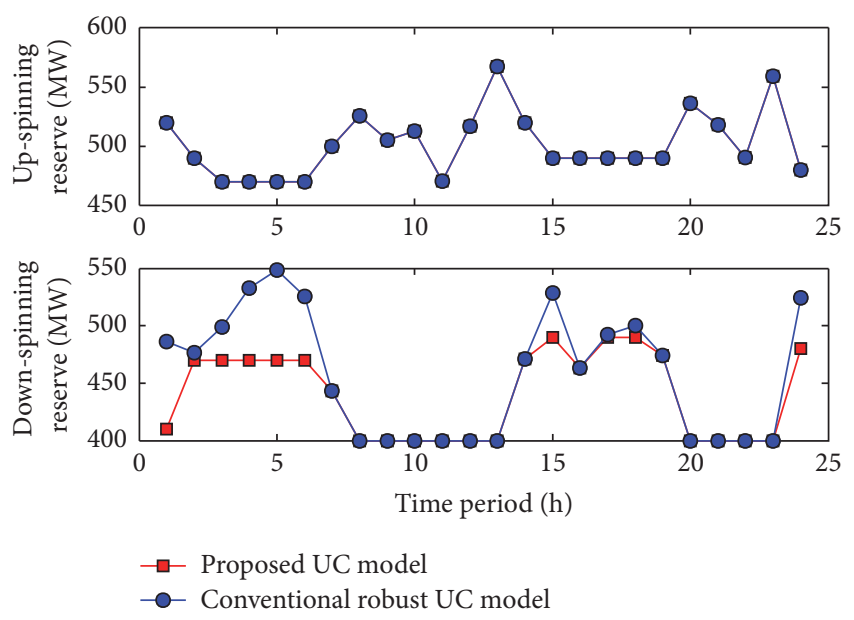

FIGURE 5: Comparison of up- and down-spinning reserve between the proposed model and conventional robust UC model.
From these figures, when the system load level is low and the wind power output is high during periods $1-6,15,18$, and 24 , since the conventional generators have insufficient downward adjustment capacity, the proposed model reduces the allowable wind power output interval to ensure the system security constraints can be satisfied within the allowable interval, while the conventional robust UC model has to stop the generators 22 and 23 to meet the system dynamic adjustment requirements.

It can be seen from Table 3 that although the proposed model considers the wind power spillage and increases the penalty cost of wind power spillage $4040.80 \$$, the start-up and shut-down cost of conventional generators reduce to $246.10 \$$, the wind power output remains unchanged, and the inexpensive generators 22 and 23 are running so that the fuel cost of conventional generators decreases to $1476.82 \$$. In addition, the uncertain interval of wind power output reduces and the spinning reserve cost of wind power requirements will reduce to $3380.34 \$$, which makes the total cost reduce to $1062.46 \$$, and the reduced proportion of the total cost is about $0.15 \%$.

\section{Conclusion}

The existing robust UC models pay more attention to the adjustment of conservativeness of the upper and lower bounds of uncertainty in each period and have a premise that wind power generation is treated as a nondispatchable resource and can be completely accommodated. In order to accurately analyze the impacts of wind power accommodation on on/off schedules and spinning reserve requirements of conventional generators and consider the transmission capacity limits, this paper establishes a novel double-level robust SCUC model with optimizable interval of uncertain wind power output to determine the optimal wind power accommodation interval by treating the boundaries of wind power prediction interval as the optimizable decision variables and not merely uncertain parameters. In addition, a two-stage iterative solution method based on BD is also presented to solve the proposed double-level model which is difficult to be solved because of the invalid dual transform in solution process caused by the coupling relation between the discrete and continuous variables.

Simulation studies on a modified IEEE 26-generator reliability test system connected to a wind farm are conducted to verify the effectiveness and advantages of the proposed model and solution method. And from the results of simulation studies, in cases where load and high penetration wind power are negatively correlated, this model can curtail wind power to maximize the overall economic efficiency of system operation, so that the optimal economic value of wind power and on/off schedules of conventional generators as well as spinning reserve requirements of systems is achieved.

However, robust optimization decisions are inevitably conservative to ensure security in the worst-case scenarios at the expense of the economy of systems operation. System operators typically prefer to make a tradeoff between robustness and economy to achieve a desired level of security. Therefore, it is important to investigate methods of reducing 
the conservativeness in the proposed double-level robust interval SCUC model. An adjustable confidence level can be introduced into the proposed robust optimization model to achieve the tradeoff between economy and security. And then the relationship between the confidence level and the uncertainty of wind power output is obtained, which is treated as the basis of adjusting the wind power prediction interval to reduce its conservativeness. These aspects will be further investigated in our subsequent researches.

\section{Appendix}

The normal double-level robust optimization model can be described as follows.

$$
\begin{array}{ll}
\max & f(x) \\
\text { s.t. } & \sum_{j} a_{i j} x_{j}+\max \sum_{j} \widehat{a}_{i j} x_{j}^{*} z_{i j} \leq b_{i}, \quad \forall i \\
& \sum_{j} z_{i j} \leq \Gamma_{i}, \quad 0 \leq z_{i j} \leq 1 \\
& \mathbf{l} \leq \mathbf{x} \leq \mathbf{u} .
\end{array}
$$

After the duality transformation, the single-level robust optimization model can be formulated as

$$
\begin{array}{ll}
\max & f(x) \\
\text { s.t. } & \sum_{j} a_{i j} x_{j}+\sum_{j} q_{i j}+\Gamma_{i} z_{i} \leq b_{i}, \quad \forall i \\
& q_{i j}+z_{i} \geq \widehat{a}_{i j} x_{j}^{*}, \quad \forall i \\
& q_{i j} \geq 0 \\
& z_{i} \geq 0 \\
& \mathbf{l} \leq \mathbf{x} \leq \mathbf{u},
\end{array}
$$

where the duality variables of decision variable $z_{i j}$ are $q_{i j}$ and $z_{i}$.

\section{Conflicts of Interest}

The authors declare that there are no conflicts of interest regarding the publication of this paper.

\section{References}

[1] B. C. Ummels, M. Gibescu, E. Pelgrum, W. L. Kling, and A. J. Brand, "Impacts of wind power on thermal generation unit commitment and dispatch," IEEE Transactions on Energy Conversion, vol. 22, no. 1, pp. 44-51, 2007.

[2] A. Tuohy, P. Meibom, E. Denny, and M. O’Malley, "Unit commitment for systems with significant wind penetration," IEEE Transactions on Power Systems, vol. 24, no. 2, pp. 592-601, 2009.

[3] L. Wu, M. Shahidehpour, and T. Li, "Stochastic security-constrained unit commitment," IEEE Transactions on Power Systems, vol. 22, no. 2, pp. 800-811, 2007.
[4] J. Wang, M. Shahidehpour, and Z. Li, "Security-constrained unit commitment with volatile wind power generation," IEEE Transactions on Power Systems, vol. 23, no. 3, pp. 1319-1327, 2008.

[5] H. Wu, M. Shahidehpour, Z. Li, and W. Tian, "Chanceconstrained day-ahead scheduling in stochastic power system operation," IEEE Transactions on Power Systems, vol. 29, no. 4, pp. 1583-1591, 2014.

[6] L. Zhang, Y. Yuan, X. Yuan, B. Chen, D. Su, and Q. Li, "Spinning reserve requirements optimization based on an improved multiscenario risk analysis method," Mathematical Problems in Engineering, vol. 2017, 2017.

[7] A. Ben-Tal and A. Nemirovski, "Robust convex optimization," Mathematics of Operations Research, vol. 23, no. 4, pp. 769-805, 1998.

[8] A. Ben-Tal and A. Nemirovski, "Robust solutions of uncertain linear programs," Operations Research Letters, vol. 25, no. 1, pp. $1-13,1999$.

[9] A. Street, F. Oliveira, and J. M. Arroyo, "Contingencyconstrained unit commitment with $\mathrm{n}-\mathrm{K}$ security criterion: A robust optimization approach," IEEE Transactions on Power Systems, vol. 26, no. 3, pp. 1581-1590, 2011.

[10] D. Bertsimas, E. Litvinov, X. A. Sun, J. Zhao, and T. Zheng, "Adaptive robust optimization for the security constrained unit commitment problem," IEEE Transactions on Power Systems, vol. 28, no. 1, pp. 52-63, 2013.

[11] R. Jiang, J. Wang, and Y. Guan, "Robust unit commitment with wind power and pumped storage hydro," IEEE Transactions on Power Systems, vol. 27, no. 2, pp. 800-810, 2012.

[12] B. Hu, L. Wu, and M. Marwali, "On the robust solution to SCUC with load and wind uncertainty correlations," IEEE Transactions on Power Systems, vol. 29, no. 6, pp. 2952-2964, 2014.

[13] C. Zhao, J. Wang, J.-P. Watson, and Y. Guan, "Multi-stage robust unit commitment considering wind and demand response uncertainties," IEEE Transactions on Power Systems, vol. 28, no. 3, pp. 2708-2717, 2013.

[14] H. Ye and Z. Li, "Robust Security-Constrained Unit commitment with recourse cost requirement," in Proceedings of the IEEE Power and Energy Society General Meeting, PESGM 2015, usa, July 2015.

[15] C. Wang, F. Liu, J. Wang, W. Wei, and S. Mei, "Risk-based admissibility assessment of wind generation integrated into a bulk power system," IEEE Transactions on Sustainable Energy, vol. 7, no. 1, pp. 325-336, 2016.

[16] W. Wu, J. Chen, B. Zhang, and H. Sun, "A robust wind power optimization method for Look-Ahead power dispatch," IEEE Transactions on Sustainable Energy, vol. 5, no. 2, pp. 507-515, 2014.

[17] S. J. Wang, S. M. Shahidehpour, S. Mokhtari, G. D. Irisarri, and D. S. Kirschen, "Short-term generation scheduling with transmission and environmental constraints using an augmented lagrangian relaxation," IEEE Transactions on Power Systems, vol. 10, no. 3, pp. 1294-1301, 1995.

[18] D. Bertsimas and M. Sim, “The price of robustness," Operations Research, vol. 52, no. 1, pp. 35-53, 2004.

[19] J. Naoum-Sawaya and S. Elhedhli, "An interior-point Benders based branch-and-cut algorithm for mixed integer programs," Annals of Operations Research, vol. 210, pp. 33-55, 2013.

[20] H. Zheng, J. Jian, R. Quan, and L. Yang, "Unit commitment algorithm based on improved Benders decomposition and perspective cut," Dianli Zidonghua Shebei/Electric Power Automation Equipment, vol. 35, no. 1, pp. 133-138, 2015. 
[21] G. Codato and M. Fischetti, "Combinatorial Benders' cuts for mixed-integer linear programming," Operations Research, vol. 54, no. 4, pp. 756-766, 2006.

[22] M. A. Ortega-Vazquez and D. S. Kirschen, "Optimizing the spinning reserve requirements using a cost/benefit analysis," IEEE Transactions on Power Systems, vol. 22, no. 1, pp. 24-33, 2007. 


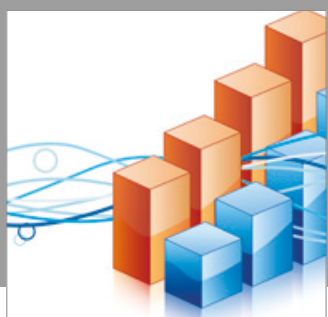

Advances in

Operations Research

vatersals

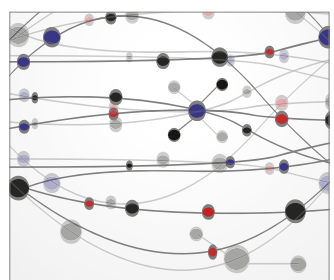

\section{The Scientific} World Journal
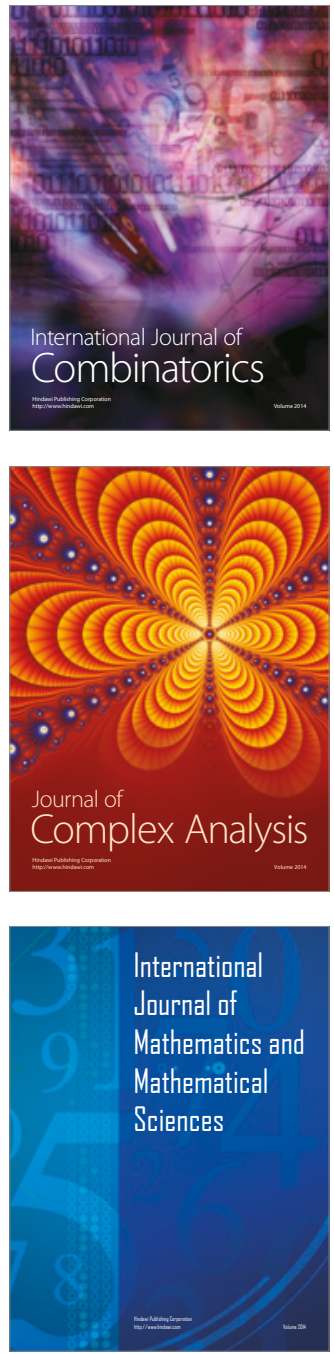
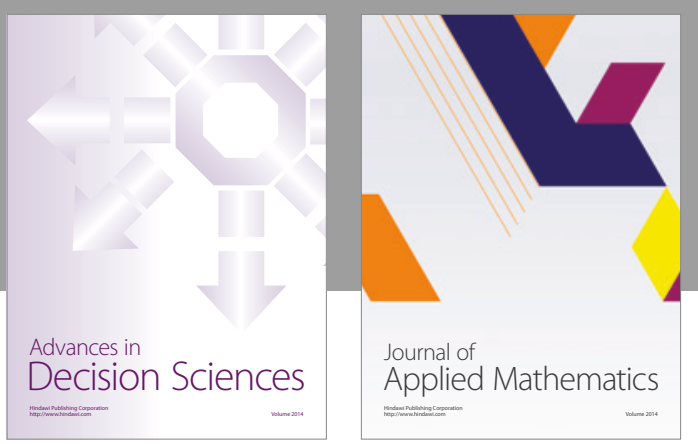

Algebra

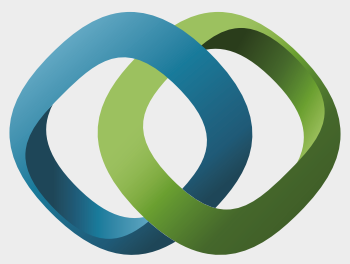

\section{Hindawi}

Submit your manuscripts at

https://www.hindawi.com
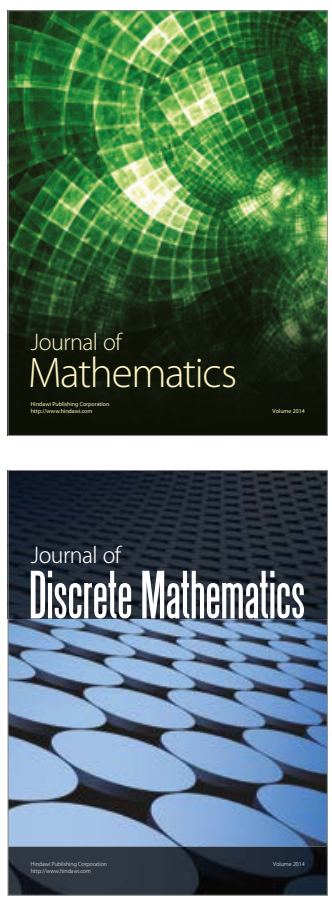

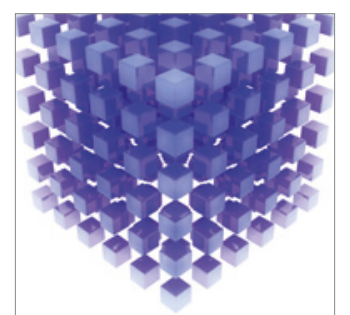

Mathematical Problems in Engineering
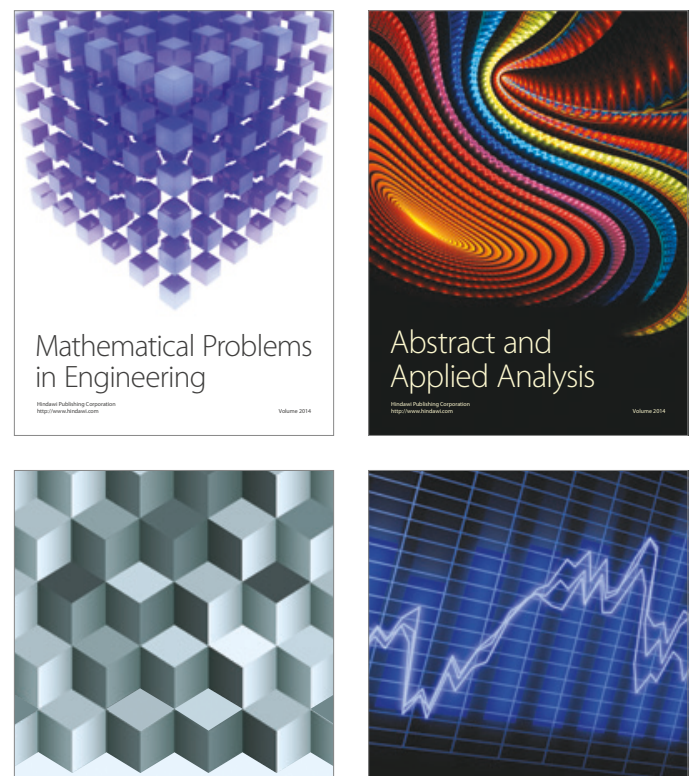

Journal of

Function Spaces

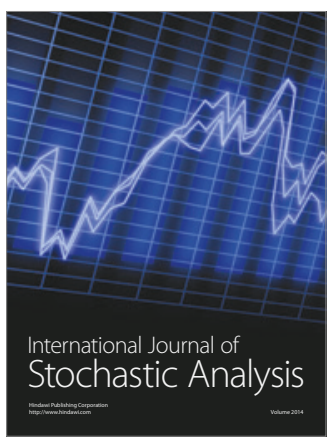

Probability and Statistics
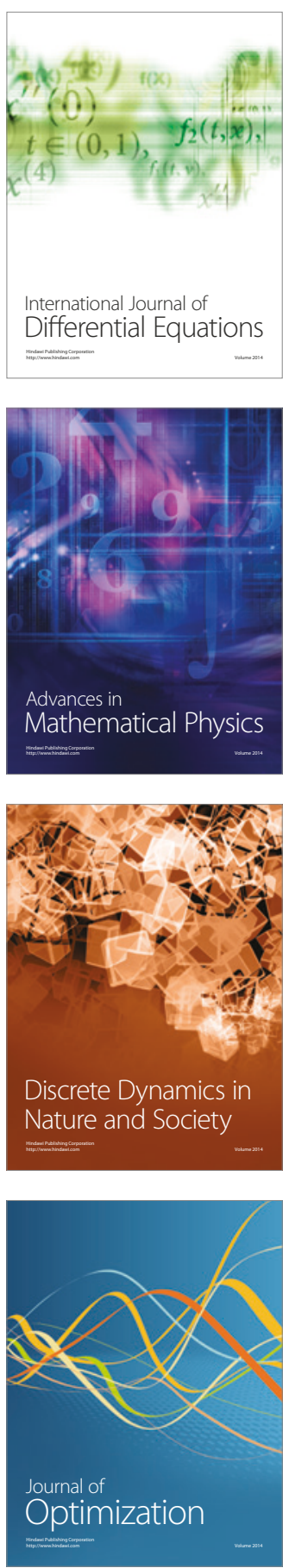